\title{
The Saturation Attack - A Bait for Twofish
}

\author{
Stefan Lucks* \\ Theoretische Informatik \\ University of Mannheim, 68131 Mannheim, Germany \\ lucks@th.informatik.uni-mannheim.de
}

\begin{abstract}
This paper introduces the notion of a "saturation attack". Consider a permutation $p$ over $w$-bit words. If $p$ is applied to all $2^{w}$ disjoint words, the set of outputs is exactly the same as the set of inputs. A saturation attack exploits this fact. The current paper applies saturation attacks on reduced-round variants of the Twofish block cipher with up to seven rounds with full whitening or eight rounds without whitening at the end (i.e., half of the cipher). The attacks take up to $2^{127}$ chosen plaintexts (half of the codebook) and are 2-4 times faster than exhaustive search. The attacks are based on key-independent distinguishers for up to six rounds of Twofish, making extensive use of saturation properties.
\end{abstract}

\section{Introduction}

Modern $b$-bit block ciphers often use permutations $p:\{0,1\}^{w} \rightarrow\{0,1\}^{w}$ with $w<b$ as building blocks. E.g., $p$ may be an S-box, a round function, or a group operation where one of the operands is constant. The constant may be unknown to the cryptanalyst, e.g. as a part of the (round) key. We regard the input for $p$ as a data channel. For the cryptanalyst, $p$ may be known or unknown, and the cryptanalysts may be unable to determine the input for $p$. A "saturation attack" is based on the idea of choosing a set of $k * 2^{w}$ plaintexts such that each of the $2^{w}$ inputs for $p$ occurs exactly $k$ times. In this case, we say that the data channel into $p$ is "saturated". A saturation attack exploits the fact that if the input for $p$ is saturated, then the output from $p$ is saturated, too.

The name "saturation attack" is new, but such attacks have been studied before. E.g., the "Square attack" is a saturation attack, developed for the block cipher Square 4. It works as well for other Square-like ciphers such as the AES candidate Crypton 1112] and the finalist Rijndael [5], which has recently been chosen as the AES. All these ciphers are 128-bit block ciphers with 8-bit data channels. The attack starts with a set of $2^{8}$ plaintexts with one saturated channel. The other 15 channels are constant. After two rounds, all 16 data channels are saturated. After three rounds, the saturation property is likely to have been lost, but the sum of all values in a data channel is zero. This allows to distinguish the three-round output from random. The best currently known attacks on Crypton [3] and Rijndael/AES [7] are extensions of the Square attack.

* Supported by German Science Foundation (DFG) grant KR 1521/3-2. 
"Miss in the middle" attacks [1] are rudimentarily related to saturation attacks, exploiting the fact that given two inputs $x \neq y$ for a permutation $p$ one gets two outputs $p(x)$ and $p(y)$ with $p(x) \neq p(y)$. Also related is the attack on "Ladder DES", based on choosing $c * 2^{32}$ distinct inputs for a 64-bit data channel and checking if all the outputs are distinct [2].

When using "higher-order differentials" [9], one chooses a certain complete set of plaintexts and, after some rounds of the cipher, predicts a key-independent property with probability one. This resembles the current approach.

This paper shows that saturation attacks are a useful tool for ciphers which are definitely not Square-like. We concentrate on the AES finalist Twofish [15]. So far, the authors of Twofish published some preliminary cryptanalytic results [16 6] themselves, a key separation property has been identified for Twofish [13. $14[8]$, and some observations on the generation of the Twofish S-Boxes and on differential cryptanalysis have been made [10].

The motivation for this research has been twofold. First, even though Twofish has not been chosen as the AES, it is (and probably will continue to be) used in practice. E.g., recent versions of popular email encryption programs, namely PGP and GnuPG [17, implement Twofish. Second, the study of saturation attacks appears to be of independent interest in cryptanalysis.

\section{$1.1 \quad$ Notation}

We will use the notion of a "multiset" to describe a $w$-bit data channel. A multiset with $k * 2^{w}$ entries is "saturated" if every value in $\{0,1\}$ w is found exactly $k$ times in the multiset. If $k=1$, a saturated multiset is the set $\{0,1\}^{w}$.

In the context of this paper, a data channel is always 32 bits wide, and we call a value in a data channel a "word". We interchangeably view a word $x$ as a 32 -bit string $x=\left(x_{31}, \ldots, x_{0}\right) \in\{0,1\}^{32}$ and as an unsigned integer $x=\sum_{i} x_{i} * 2^{i}$. The addition of values in a data channel is thus addition mod $2^{32}$. We write " $x \lll b$ " for the rotation of the word $x$ by $b$ bits to the left, and " $x \ggg b$ " for rotation to the right. E.g. $(x \lll b) \ggg b=x$ for all $x$ and $b$, and $\left(x_{31}, x_{30}, \ldots, x_{1}, x_{0}\right) \lll 1=\left(x_{30}, \ldots, x_{1}, x_{0}, x_{31}\right)$. LSB $(x)=x$ mod 2 denotes the "least significant bit (LSB)" of $x$, and $\operatorname{LSB}^{1}(x)=\operatorname{LSB}(x \operatorname{div} 2)$ denotes the 2nd-least significant bit. Similarly, we define the "most significant bit (MSB)": $\operatorname{MSB}(x)=\operatorname{LSB}(x \lll 1)$. If the multiset $M$ denotes a data channel, the bits at the LSB-position of $M$ are "balanced" if $\bigoplus_{m \in M} \operatorname{LSB}(m)=0$. It turns out to be useful to also consider "semi-saturated" data channels. The multiset $M$ is semi-saturated if one bit of $M$ is constant and each of the $2^{31}$ remaining values for $M$ appears exactly $2 k$ times in $M$.

\section{A Description of Twofish}

In this section, we describe the structure of Twofish. We omit many details, concentrating on the properties of Twofish which are relevant for our attack. 


\subsection{The Main Operations of Twofish}

Twofish is based on the following operations:

Whitening. Decompose a 128-bit text block into words $a_{0}, \ldots, a_{3} \in\{0,1\}^{32}$.

The Twofish whitening operation is the XOR of four key words $K_{j+\delta} \in$ $\{0,1\}^{32}$ to the words $a_{j}: b_{j}:=a_{j} \oplus K_{j+\delta}$ for $j \in\{0, \ldots, 3\}$, see Figure 1.

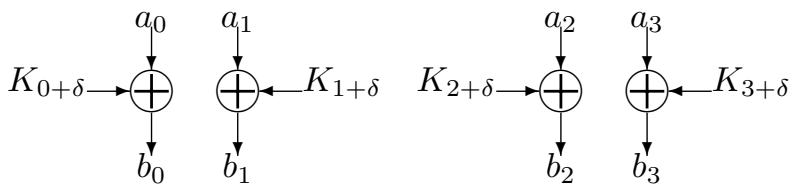

Fig. 1. The Twofish Whitening Operation.

Application of the round function. To compute the $i$-th round function $F_{i}$, use a pair $(a, b) \in\left(\{0,1\}^{32}\right)^{2}$ as the input and and compute a pair $\left(a^{\prime}, b^{\prime}\right)=$ $F_{i}(a, b) \in\left(\{0,1\}^{32}\right)^{2}$. The round function $F_{i}$ is defined by two round keys $K_{2 i+2}$ and $K_{2 i+3}$ and two functions $G_{1}, G_{2}:\{0,1\}^{32} \rightarrow\{0,1\}^{32}$, see Fig. 2.

$$
a^{\prime}:=G_{1}(a)+G_{2}(b)+K_{2 i+2}, \quad \text { and } \quad b^{\prime}:=G_{1}(a)+2 G_{2}(b)+K_{2 i+3},
$$

The functions $G_{1}$ and $G_{2}$ are key-dependent, but do not depend on $i$. Given the round function's results $a^{\prime}$ and $b^{\prime}$, the remaining two words $c, d \in\{0,1\}^{32}$ come into play:

$$
x:=\left(a^{\prime} \oplus c\right) \ggg 1, \quad \text { and } \quad y:=b^{\prime} \oplus(d \lll 1) .
$$

Except for the rotate operations, Twofish works like a Feistel cipher.

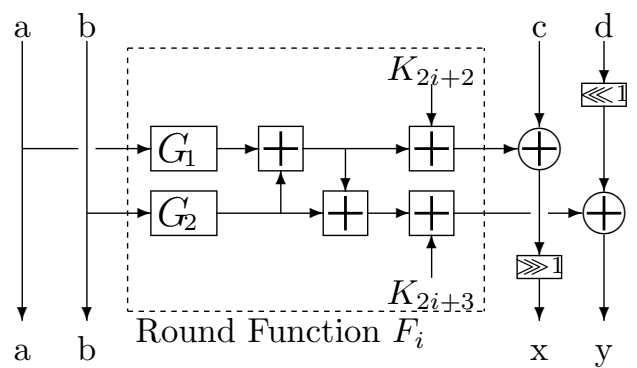

Fig. 2. The Application of the Twofish Round Function.

Our attack greatly depends on the functions $G_{1}$ and $G_{2}$ to be permutations over $\{0,1\}^{32}$. Actually, $G_{2}(x)=G_{1}(x \lll 8)$. Apart from that, the internal structure of $G_{1}$ and $G_{2}$ is not relevant for us.

The swap. Replace $(a, b, c, d) \in\left(\{0,1\}^{32}\right)^{4}$ by $(c, d, a, b)$. See Figure 3 


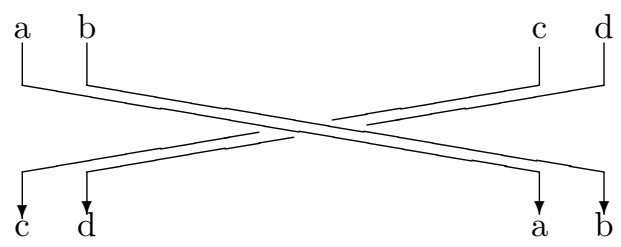

Fig. 3. The Twofish Swap.

\subsection{The Basic Structure of Twofish}

Twofish uses a 16-round Feistel structure with two additional one-bit rotates in each round, pre-whitening before the first round and post-whitening after the last round. Twofish works as follows:

1. Generate the key-dependent S-boxes, which define the functions $G_{1}$ and $G_{2}$.

2. Generate four subkey words $K_{0}, \ldots K_{3} \in\{0,1\}^{32}$ for the pre-whitening, two subkey words $K_{2 i+2}, K_{2 i+3}$ for each round and another four subkey words $K_{36}, \ldots, K_{39}$ for the post-whitening.

3. Given a plaintext block, do the pre-whitening.

4. For $i:=1$ to 15 do: (a) Apply the round function $F_{i}$.

(b) Do the swap.

5. Apply the last round function $F_{16}$ (no swap in the final round).

6. Do the post-whitening.

The first two of the above steps constitute the "key schedule" described below. Note that we can obviously generalise the Twofish structure to $r$ rounds, where the loop in step 4 is iterated $r-1$ times.

\subsection{The Twofish Key Schedule}

A Twofish key consists of 128, 192, or 256 bit1: $2 k$ words $M_{0}, \ldots, M_{2 k-1} \in$ $\{0,1\}^{32}$ with $k \in\{2,3,4\}$, organised as two vectors $M_{e}=\left(M_{0}, M_{2}, \ldots, M_{2 k-2}\right)$ and $M_{o}=\left(M_{1}, M_{3}, \ldots, M_{2 k-1}\right)$. A third vector $S=\left(S_{0}, S_{1}, \ldots, S_{k-1}\right)$ is derived from $M_{e}$ and $M_{o}$ by using techniques from the theory of Reed-Solomon codes. Given any two of the three vectors $M_{e}, M_{o}$ and $S$, the third one is easy to find.

With these three vectors, the "three halves of a Twofish key", we can do the first two steps of the structure described above:

1. The vector $S$ determines the internal S-boxes and thus the functions $G_{1}$ and $G_{2} . S$ is a $k$-word vector, while the key consists of $2 k$ words or $64 k$ bit.

2. The 40 subkey words $K_{0}, \ldots, K_{39}$ are defined by using functions $h_{e}$ and $h_{o}$ and by doing 20 "subkey generation" steps $(j \in\{0, \ldots, 19\})$ :

$$
\begin{array}{ll}
A_{j}:=h_{e}\left(j, M_{e}\right) ; & K_{2 j}:=A_{j}+B_{j} ; \\
B_{j}:=h_{o}\left(j, M_{o}\right) ; & K_{2 j+1}:=\left(A_{j}+2 B_{j}\right) \lll 9 .
\end{array}
$$

$\overline{1}$ These are the three generic key lengths of Twofish. Other keys of less than 256 bit are padded to the next generic length by appending zeros. 


\section{Distinguishers for Twofish}

Given a well-chosen set of plaintexts, we describe how to distinguish reducedround versions of Twofish from random permutations.

\subsection{A Four-Round Distinguisher}

Consider $2^{32}$ plaintexts $\left(\alpha_{0}, \alpha_{1}, A, \alpha_{3}\right)$, where $\alpha_{0}, \alpha_{1}$, and $\alpha_{3}$ are three arbitrary 32-bit constants and $A$ is the set of all $2^{32}$ words. The pre-whitening changes this set of texts to $\left(\beta_{0}, \beta_{1}, A, \beta_{3}\right)$ with new constants $\beta_{i}$.

Given this set of texts as the input for the first round, the input for the round function $F_{1}$ is constant: $\left(\beta_{0}, \beta_{1}\right)$. By $\left(\gamma_{0}, \gamma_{1}\right)$ we denote the output of $F_{1}$, which then generates the texts $\left(\beta_{0}, \beta_{1}, A, \gamma_{3}\right)$ with $\gamma_{3}=\left(\beta_{3} \lll 1\right) \oplus \gamma_{1}$. (Note that $A=\left\{a_{i}\right\}=\left\{\left(a_{i} \oplus \gamma_{0}\right) \ggg 1\right\}$.) The swap changes these texts to $\left(A, \gamma_{3}, \beta_{0}, \beta_{1}\right)$.

In the second round, the $2^{32}$ inputs for the round function are $\left(A, \gamma_{3}\right)$. The round function generates the pairs $\left(b_{i}, c_{i}\right)$ with $b_{i}=G_{1}\left(a_{i}\right)+G_{2}\left(\gamma_{3}\right)+K_{6}$ and $c_{i}=$ $G_{1}\left(a_{i}\right)+2 G_{2}\left(\gamma_{3}\right)+K_{7}$ for $a_{i} \in A$. The sets $B=\left\{b_{i}\right\}$ and $C=\left\{c_{i}\right\}$ are saturated, just like $A$. Applying the round function here means XORing the constant $\beta_{0}$ to the values of $B$, followed by a rotation, and XORing $\beta_{1} \lll 1$ to $C$. Neither operation changes the saturated sets $B$ and $C$. We get $2^{32}$ texts $\left(A, \gamma_{3}, B, C\right)$, where $A, B$, and $C$ are saturated. By the swap, we get texts $\left(B, C, A, \gamma_{3}\right)$.

The $2^{32}$ inputs for the third round function are of the form $(B, C)$ with saturated $B$ and $C$. Since both $G_{1}$ and $G_{2}$ are permutations, $G_{1}\left(b_{i}\right) \neq G_{1}\left(b_{j}\right)$ and $G_{2}\left(c_{i}\right) \neq G_{2}\left(c_{j}\right)$ for $b_{i}, b_{j} \in B, c_{i}, c_{j} \in C$, and $i \neq j$. Let $d_{i}=G_{1}\left(b_{i}\right)+G_{2}\left(c_{i}\right)+K_{8}$ and $e_{i}=G_{1}\left(b_{i}\right)+2 G_{2}\left(c_{i}\right)+K_{9}$. The $2^{32}$ outputs of the round function are of the form $(D, E)$, with the multisets $D=\left\{d_{i} \mid 0 \leq i<2^{32}\right\}$ and $E=\left\{e_{i} \mid 0 \leq i<2^{32}\right\}$. Neither $D$ nor $E$ is likely to be saturated. However, we are still able to observe a weaker property: Since $\sum_{b_{i} \in B} b_{i}=\sum_{c_{i} \in C} c_{i}=\sum_{0 \leq i<2^{32}} i \equiv 2^{31} \bmod 2^{32}$ :

$$
\begin{gathered}
\sum_{0 \leq i<2^{32}} d_{i} \equiv 2^{31}+2^{31}+2^{32} * K_{8} \equiv 0\left(\bmod 2^{32}\right), \\
\sum_{0 \leq i<2^{32}} e_{i} \equiv 2^{31}+2 * 2^{31}+2^{32} * K_{9} \equiv 2^{31}\left(\bmod 2^{32}\right),
\end{gathered}
$$

thus $\sum d_{i} \equiv \sum e_{i} \equiv 0(\bmod 2)$ - i.e., the LSBs of $D$ and $E$ are balanced.

Applying the round function means to evaluate $2^{32}$ pairs $\left(f_{i}, g_{i}\right)$ with $f_{i}=$ $f_{i}^{\prime} \ggg 1, f_{i}^{\prime}=a_{i} \oplus d_{i}$, and $g_{i}=\left(\gamma_{3} \lll 1\right) \oplus e_{i}$. Define the multisets $F=\left\{f_{i}\right\}$, $F^{\prime}=\left\{f_{i}^{\prime}\right\}$, and $G=\left\{g_{i}\right\}$. We observe: The bits at the LSB-positions of both $F^{\prime}$ and $G$ are balanced, and, due to the rotate, the bits at the MSB-position of $F$ are balanced. Hence, the third round generates $2^{32}$ texts of the form $(B, C, F, G)$, which are then swapped to $(F, G, B, C)$.

The multisets $(F, G)$ of inputs for the fourth round function $F_{4}$ are balanced. We write $(?, ?)$ for the outputs. Applying $F_{4}$ gives us $2^{32}$ texts $(F, G, ?, ?)$. After the swap, we get $(?, ?, F, G)$, where one bit in each $F$ and $G$ is balanced. 
Figure 4 describes graphically, how the distinguisher works. Having chosen $2^{32}$ plaintexts, we can check the balancedness of the ciphertext bits at the two positions determined by the MSB of $F$ and the LSB of $G$. Whatever the keys are, four rounds of Twofish always pass this test - even the post-whitening cannot destroy the balancedness. But a random permutation only passes this test with about a $25 \%$ probability.

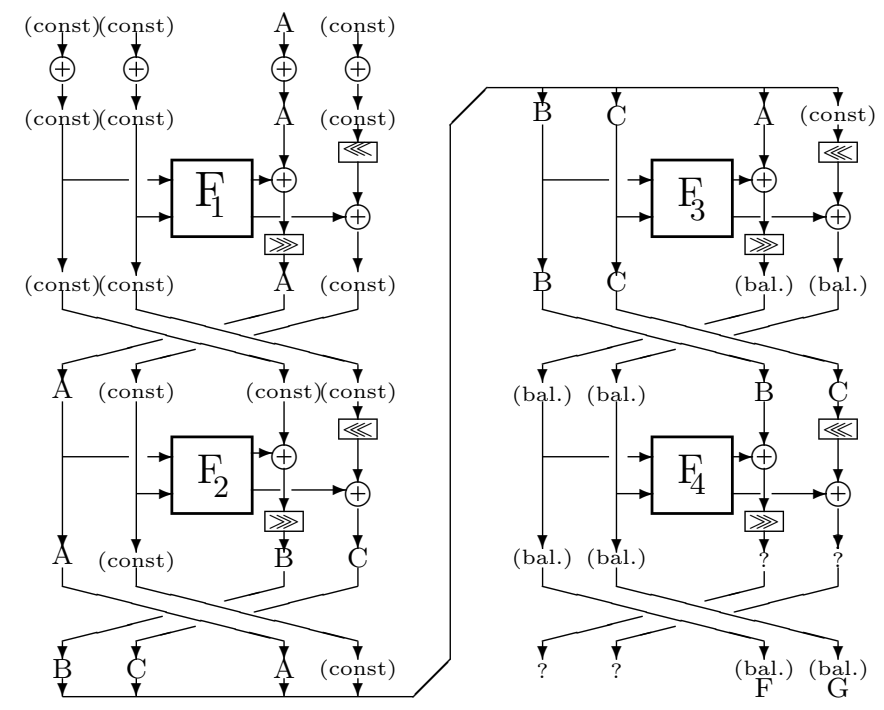

Fig. 4. The Four-Round Distinguisher from Section 3.1

\subsection{Another Four-Round Distinguisher}

Our second distinguisher works quite similarly to the first one. We start with $2^{32}$ plaintexts of the form $\left(\alpha_{0}, \alpha_{1}, \alpha_{2}, A\right)$ with arbitrary constants $\alpha_{i}$. The prewhitening changes the constants and we get texts $\left(\beta_{0}, \beta_{1}, \beta_{2}, A\right)$. After the first round, including the swap, these are $\left(\gamma_{2}, A, \beta_{0}, \beta_{1}\right)$.

In the second round, the inputs to the round function are of the form $\left(\gamma_{2}, A\right)$, where $A=\{0,1\}^{32}$ is a saturated set and $\gamma_{2}$ is constant. The round function generates the pairs $\left(b_{i}, c_{i}\right)$ with $b_{i}=G_{1}\left(\gamma_{2}\right)+G_{2}\left(a_{i}\right)+K_{6}$ and $c_{i}=G_{1}\left(\gamma_{2}\right)+2 G_{2}\left(a_{i}\right)+K_{7}$ for $a_{i} \in A$. Now the set $B=\left\{b_{i}\right\}$ is saturated like $A$, but the multiset $C^{*}=\left\{c_{i}\right\}$ isn't. Instead, it is semi-saturated with a constant $\operatorname{LSB}\left(c_{i}\right)=\gamma^{*} \in\{0,1\}$ for all $c_{i} \in C^{*}: \gamma^{*}=\operatorname{LSB}\left(G_{1}\left(\gamma_{2}\right)\right) \oplus \operatorname{LSB}\left(K_{7}\right)$. We apply the round function by adding some constants to the elements of $B$ and $C^{*}$, and by then rotating the elements of $B$. The results are a saturated set $B$ and a semi-saturated set $C^{*}$, as before. After the swap, we have texts of the form $\left(B, C^{*}, \gamma_{2}, A\right)$.

In the third round the $2^{32}$ inputs for the round function are of the form $\left(B, C^{*}\right)$. Consider the round function's outputs $\left(d_{i}, e_{i}\right)$ with $d_{i}=G_{1}\left(b_{i}\right)+$ 
$G_{2}\left(c_{i}\right)+K_{8}$ and $e_{i}=G_{1}\left(b_{i}\right)+2 G_{2}\left(c_{i}\right)+K_{9}$. Since $B=\left\{b_{i}\right\}$ is saturated, so is $\left\{G_{1}\left(b_{i}\right)\right\}$, and especially

$$
\sum_{0 \leq i<2^{32}} G_{1}\left(b_{i}\right) \equiv 0(\bmod 2) .
$$

Since $C^{*}$ is semi-saturated, it has $2^{31}$ different values, each repeated exactly twice. The same holds for the $2^{32}$ values $G_{2}\left(c_{i}\right)$ (with $c_{i} \in C^{*}$ ), hence

$$
\sum_{0 \leq i<2^{32}} G_{2}\left(c_{i}\right) \equiv 0(\bmod 2)
$$

Thus, both multisets $D=\left\{d_{i}\right\}$ and $E=\left\{e_{i}\right\}$ are balanced:

$$
\sum_{0 \leq i<2^{32}} d_{i}=\sum_{i} G_{1}\left(b_{i}\right)+\sum_{i} G_{2}\left(c_{i}\right)+2^{32} * K_{8} \equiv 0(\bmod 2)
$$

and

$$
\sum_{0 \leq i<2^{32}} e_{i}=\sum_{i} G_{1}\left(b_{i}\right)+2 * \sum_{i} G_{2}\left(c_{i}\right)+2^{32} * K_{9} \equiv 0(\bmod 2) .
$$

By applying the round function and swapping, we get $2^{32}$ texts of the form $\left(F, G, B, C^{*}\right)$. The bits at the LSB-position of $G$ are balanced, as are the bits at the MSB-position of $F$ (due to the one-bit rotate). The fourth round makes this $(F, G, ?, ?)$, and if we do the swap we get texts of the form $(?, ?, F, G)$.

A random permutation passes the corresponding test only with a probability of about 0.25 .

\subsection{An Extension to Five Rounds}

Next, we show how to extend the distinguisher from Section 3.2 to five rounds. Let $\alpha$ an arbitrary 32-bit constant and $c^{*}$ an arbitrary 1-bit constant. We choose all $2^{95}$ plaintexts of the form $\left(\alpha, a_{i}, b_{j}, c_{k}\right)$, with $c_{i} \operatorname{div} 2^{31}=c^{*}$. We write $\left(\alpha, A, B, C^{+}\right)$for these $2^{95}$ texts. Note that the multisets $A$ and $B$ are saturated and the multiset $C^{+}$is semi-saturated. The pre-whitening changes the constant $\alpha$ to $\beta$, and the constant $c^{*}$ to $\gamma^{*}$, but leaves $A$ and $B$ saturated and $C^{+}$semi-saturated with a constant MSB. We still have $2^{95}$ distinct input texts $\left(\beta, A, B, C^{+}\right)$for the first round.

Let $\left(e_{i}, f_{i}\right)=F_{1}\left(\beta, a_{i}\right)$ with $a_{i} \in A$. We can write $e_{i}=\beta_{e}+G_{2}\left(a_{i}\right)$ and $f_{i}=\beta_{f}+2 G_{2}\left(a_{i}\right)$, for some constants $\beta_{e}, \beta_{f}$. Hence the outputs of $F_{1}$ consist of pairs $\left(E, F^{*}\right)$ with saturated $E$ and semi-saturated $F^{*}$. Set $\beta^{*}=f_{i} \bmod 2$ for the constant LSB of the values $f_{i} \in F^{*}$.

For every value $a_{i} \in A$ there are $2^{63}$ pairs $\left(b_{i}, c_{i}\right)$ with a constant bit $\gamma^{*}=$ $c_{i} \operatorname{div} 2^{31}=\operatorname{MSB}\left(c_{i}\right)$. We can fix any constants $\gamma_{2}, \gamma_{3} \in\{0,1\}^{32}$ with $\gamma_{3} \bmod 2=$ $\gamma^{*} \oplus \beta^{*}$ and find pairs $\left(b_{i}, c_{i}\right)$ in $\left(B, C^{+}\right)$such that $\left(e_{i} \oplus b_{i}\right) \ggg 1=\gamma_{2}$ and $f_{i} \oplus\left(c_{i} \lll 1\right)=\gamma_{3}$ holds for every $a_{i}$. (Note that the MSB of $c_{i}$ is the LSB of $c_{i} \lll 1$.) 
Now the $2^{95}$ input texts $\left(\beta, A, B, C^{+}\right)$can be separated into $2^{63}$ disjoint groups of $2^{32}$ texts, determined by the pair $\left(\gamma_{2}, \gamma_{3}\right)$ of constants, such that after applying the first round functions all texts in the same group are of the form $\left(\beta, A, \gamma_{2}, \gamma_{3}\right)$. The swap changes these to $\left(\gamma_{2}, \gamma_{3}, \beta, A\right)$.

For each such group, applying the four-round distinguisher from Section 3.2 would result in a set of $2^{32}$ ciphertexts $(?, ?, F, G)$, where the ciphertext bits at the LSB-position of $G$ and at the MSB-position of $F$ are balanced. Now, we do not know which ciphertexts belong into which group, but if these bits for each group are balanced, then so are all $2^{95}$ such bits. Five rounds of Twofish always pass this test, while a random permutation passes it with about $25 \%$ probability.

The same technique can also be applied to the distinguisher from Section 3.1. Here, we need $2^{96}$ plaintexts of the form $(\alpha, A, B, C)$ with constant $\alpha$. A random permutation passes the corresponding test with about $25 \%$ probability.

\subsection{An Extension to Six Rounds}

To attack six rounds, we choose $2^{127}$ plaintexts $\left(a_{i}, b_{i}, c_{i}, d_{i}\right)$, (half of the codebook (!)), where $b_{i} \operatorname{div} 2^{31}=\operatorname{MSB}\left(b_{i}\right)$ is fixed to an arbitrary constant. Our plaintexts are of the form $\left(A, B^{+}, C, D\right)$, where $A, B$, and $D$ are saturated multisets, and $B^{+}$is a semi-saturated one.

Our choice of plaintexts ensures that for each of the $2^{63}$ left-side pairs $\left(a_{i}, b_{i}\right)$, all $2^{64}$ right-side pairs $\left(c_{i}, d_{i}\right)$ exist. Neither the pre-whitening nor the application of the first round function change this property. By the swap we get $2^{127}$ texts $\left(C, D, A, B^{+}\right)$as the input for the second round. For each 32-bit constant $\alpha$ we get a group of $2^{95}$ texts $\left(\alpha, D, A, B^{+}\right)$. These are $2^{32}$ disjoint groups which are the kind of input we need for the 5-round distinguisher.

After six rounds of Twofish, we get $2^{127}$ ciphertexts $(?, ?, F, G)$ with balanced bits at two positions. A random permutation does satisfy this with about $25 \%$ probability.

\subsection{Distinguishers: Summary}

In Table 1 we summarise the distinguishers we have found. We describe which section the distinguisher was described in, the number $r$ of Twofish rounds the attack works for, the chosen plaintexts required (how they look like and how many we need), and the probability for a random permutation to pass the test. All tests are one-sided, i.e. $r$ rounds of Twofish pass the test with probability 1.

\section{Finding the Key}

In modern cryptanalysis, one often uses a distinguisher for some rounds of a product cipher to find the key: Guess some key bits for one or more additional rounds and exploit the distinguishing property to falsify wrong key guesses. This is what we do below, concentrating on using the six-round distinguisher. 
Table 1. Distinguishers for Twofish.

\begin{tabular}{|c|c|c|c|c|}
\hline Section & Rounds & \multicolumn{2}{|c|}{ Chosen Plaintexts } & Probability \\
& $r$ & Form & Number & \\
\hline \hline 3.1 & 4 & $\left(\alpha_{0}, \alpha_{1}, A, \alpha_{3}\right)$ & $2^{32}$ & $25 \%$ \\
\hline 3.2 & 4 & $\left(\alpha_{0}, \alpha_{1}, \alpha_{2}, A\right)$ & $2^{32}$ & $25 \%$ \\
\hline 3.3 & 5 & $\left(\alpha, A, B, C^{+}\right)$ & $2^{95}$ & $25 \%$ \\
\hline 3.3 & 5 & $(\alpha, A, B, C)$ & $2^{96}$ & $25 \%$ \\
\hline 3.4 & 6 & $\left(A, B^{+}, C, D\right)$ & $2^{127}$ & $25 \%$ \\
\hline
\end{tabular}

\subsection{The Basic Technique}

Consider seven rounds of Twofish. Let $2^{127}$ plaintexts be chosen as required for the six-round distinguisher. After six rounds (including the swap), we have $2^{127}$ text quadruples $\left(a_{i}, b_{i}, c_{i}, d_{i}\right)$ of 32 -bit words. We have two distinguishing properties: the bits at the LSB-position of the words $d_{i}$ are balanced, and the bits at the MSB-position of the $c_{i}$-words are balanced. We start with concentrating on the first distinguishing property.

The XOR over the $2^{127} \operatorname{LSBs} \delta_{i}=d_{i} \bmod 2$ of $d_{i}$ is:

$$
\delta^{*}=\bigoplus_{0 \leq i<2^{127}} \delta_{i}=0
$$

After seven rounds, we have $2^{127}$ ciphertext quadruples $\left(w_{i}, x_{i}, y_{i}, z_{i}\right)$, and we cannot count on balanced ciphertext bits. Note that the seventh round uses the keys $K_{16}$ and $K_{17}$, and the post-whitening keys denoted by $K_{18}, \ldots, K_{21}$. Figure 5 visualises the last round, including the post-whitening.

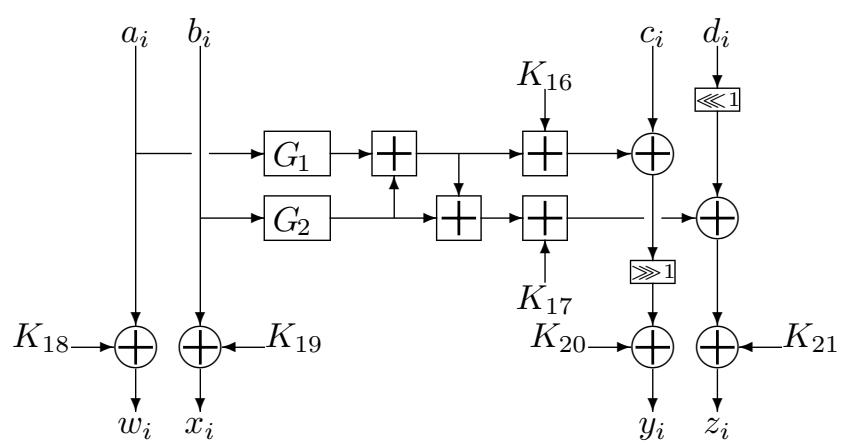

Fig. 5. The Seventh Round and the Post-Whitening. 
Rewrite $\delta_{i}$ by $\delta_{i}=\delta_{w, i} \oplus\left(\delta_{w, i}^{\mathrm{c}} * \operatorname{LSB}\left(K_{17}\right)\right) \oplus \delta_{x, i} \oplus \delta_{z, i} \oplus \operatorname{LSB}^{1}\left(K_{17}\right)$ with

$$
\begin{aligned}
\delta_{w, i} & =\operatorname{LSB}^{1}\left(G_{1}\left(w_{i} \oplus K_{18}\right)\right) \\
\delta_{w, i}^{\mathrm{c}} & =\operatorname{LSB}\left(G_{1}\left(w_{i} \oplus K_{18}\right)\right), \\
\delta_{x, i} & =\operatorname{LSB}\left(G_{2}\left(x_{i} \oplus K_{19}\right)\right), \\
\delta_{z, i} & =\operatorname{LSB}^{1}\left(z_{i}\right) \oplus \operatorname{LSB}^{1}\left(K_{21}\right) .
\end{aligned}
$$

The necessity to consider the 2nd-least significant bits $\operatorname{LSB}^{1}(\ldots)$ is due to the last round's one-bit rotate: $\operatorname{LSB}\left(d_{i}\right)=\operatorname{LSB}^{1}\left(d_{i} \lll 1\right)$. Note that the value $\left(\delta_{w, i}^{\mathrm{c}} * \operatorname{LSB}\left(K_{17}\right)\right) \in\{0,1\}$ specifically deals with the carry bit generated at the LSB-position.

We can evaluate the bit $\delta_{i}$ by partial decryption. Since we are rather interested in the bit $\delta^{*}=\bigoplus_{i} \delta_{i}$, we rewrite $\delta^{*}$ by

$$
\delta^{*}=\delta_{w}^{*} \oplus\left(\delta_{w}^{* \mathrm{c}} * \operatorname{LSB}\left(K_{17}\right)\right) \oplus \delta_{x}^{*} \oplus \delta_{z}^{*}
$$

with

$$
\begin{aligned}
\delta_{w}^{*} & =\bigoplus_{0 \leq i<2^{127}} \delta_{w, i}=\bigoplus_{i}\left(\operatorname{LSB}^{1}\left(G_{1}\left(w_{i} \oplus K_{18}\right)\right)\right) \\
\delta_{w}^{* \mathrm{c}} & =\bigoplus_{0 \leq i<2^{127}} \delta_{w, i}^{\mathrm{c}}=\bigoplus_{i}\left(\operatorname{LSB}\left(G_{1}\left(w_{i} \oplus K_{18}\right)\right)\right) \\
\delta_{x}^{*} & =\bigoplus_{0 \leq i<2^{127}} \delta_{x, i}=\bigoplus_{i} \operatorname{LSB}\left(G_{2}\left(x_{i} \oplus K_{19}\right)\right) \\
\delta_{z}^{*} & =\bigoplus_{0 \leq i<2^{127}} \delta_{z, i}=\bigoplus_{i}\left(\operatorname{LSB}^{1}\left(z_{i}\right) \oplus \operatorname{LSB}^{1}\left(K_{21}\right)\right)=\bigoplus_{i} \operatorname{LSB}^{1}\left(z_{i}\right)
\end{aligned}
$$

Assume an adversary to know (or to have guessed) the S-boxes, i.e., to know the functions $G_{1}$ and $G_{2}$. Given $2^{127}$ ciphertexts $\left(w_{i}, x_{i}, y_{i}, z_{i}\right)$, we can compute the bits $\delta_{w}^{*}, \delta_{w}^{* \mathrm{c}}, \delta_{x}^{*}$ and $\delta_{z}^{*}$ independently.

For $\delta_{z}^{*}$ just count how often one of the $2^{127} \operatorname{bits} \operatorname{LSB}^{1}\left(z_{i}\right)$ is one $-\delta_{z}^{*}$ is just this number (modulo 2). Regarding $\delta_{x}^{*}$ we just need to consider all words $x_{i}$ which appear an odd time as a part of a ciphertext $\left(w_{k}, x_{i}, y_{k}, z_{k}\right)$. These are at most $2^{32}$ words $x_{i}$. Given these and the key word $K_{19}$ we just count mod 2 how often $\operatorname{LSB}\left(G_{2}\left(x_{i} \oplus K_{19}\right)\right)$ is one. Computing $\delta_{w}^{*}$ and $\delta^{* c}$ can be done similarly.

\subsection{Attacking Seven Rounds with Full Whitening}

In this section we describe and analyse the seven-round attack. Consider the following algorithm.

0. Choose $2^{127}$ plaintexts as required for the six-round distinguisher. Ask for the corresponding ciphertexts $\left(w_{i}, x_{i}, y_{i}, z_{i}\right), 0 \leq i<2^{127}$.

1. For all $W \in\{0,1\}^{32}$ : count $(\bmod 2)$ the ciphertexts $\left(W, x_{i}, y_{i}, z_{i}\right)$.

2. For all $X \in\{0,1\}^{32}$ : count $(\bmod 2)$ the ciphertexts $\left(w_{i}, X, y_{i}, z_{i}\right)$. 
3. Evaluate $\delta_{z}^{*}$ by counting the bits $\operatorname{LSB}^{1}\left(z_{i}\right)$ of the words $z_{i}$.

4. Guess the key-dependent vector $S$, defining the functions $G_{1}$ and $G_{2}$.

5. Consider the $2^{32}$ one-bit counters for ciphertexts $\left(w_{i}, X, y_{i}, z_{i}\right)$. For every key $K_{19} \in\{0,1\}^{32}$ evaluate $\delta_{x}^{*}$. Write $\delta_{x}^{*}\left(K_{19}\right)$ for the results.

6. Consider the $2^{32}$ one-bit counters for ciphertexts $\left(W, x_{i}, y_{i}, z_{i}\right)$. For every choice of $K_{18}$ evaluate $\delta_{w}^{*}$ and $\delta_{w}^{* c}$. Write $\delta_{w}^{*}\left(K_{18}\right)$ and $\delta_{w}^{* c}\left(K_{18}\right)$ for the results.

7. Check the subkey triple $\left(\operatorname{LSB}\left(K_{17}\right), K_{18}, K_{19}\right) \in\{0,1\} \times\{0,1\}^{32} \times\{0,1\}^{32}$. It is is "valid for $S$ " if and only if

$$
\delta_{w}^{*}\left(K_{18}\right) \oplus\left(\delta_{w}^{* c}\left(K_{18}\right) * \operatorname{LSB}\left(K_{17}\right)\right) \oplus \delta_{x}^{*}\left(K_{19}\right)=\delta_{z}^{*} .
$$

How expensive is this algorithm? Let $K \in\{128,192,256\}$ be the key size, i.e. each of the "key halfs" $M_{e}, M_{o}$, and $S$ consists of $K / 64 \in\{2,3,4\}$ words. Obviously, we need $2^{127}$ chosen plaintexts, half of the codebook. Each of the steps 13 requires $2^{127}$ very simple operations (incrementing a one-bit counter). Step 4 requires to guess $K / 2$ key bits. Each of the steps $[5]$ is repeated $2^{K / 2}$ times:

- Note that we can compute and store a table with $2^{32}$ entries for the function $G_{1}$. Then for each key $K_{19}$, step $\left[5\right.$ requires an average of $2^{31}$ very simple operations (essentially table look-ups).

- Since $G_{2}(x)=G_{1}(x \ggg 8)$, we can reuse the table for $G_{1}$ for the function $G_{2}$. Then for Each key $K_{18}$, step [6 requires $2 * 2^{32}$ very simple operations, on the average.

Hence this algorithm requires about $3 * 2^{(K / 2)+64}$ "very simple" operations. Since such an operation is much faster than a single Twofish encryption, the attack is much faster than exhaustive key search for $K \geq 128$.

The algorithm allows us to filter out half of the keys. (I.e., about $50 \%$ of all random permutations pass the test.) So a key finding attack based on the algorithm has to exhaustively search the remaining half of the key space, requiring an expected number of $2^{K-2}$ Twofish encryptions.

\subsection{Attacking Eight Rounds without Post-whitening}

The previous attack can be modified to work for eight rounds of Twofish without post-whitening. Let the usual $2^{127}$ plaintext be chosen. After six rounds, including the swap, we have $2^{127}$ quadruples $\left(a_{i}, b_{i}, c_{i}, d_{i}\right)$ of 32 bit words, and

$$
\delta^{*}=0=\bigoplus_{0 \leq i<2^{127}} \operatorname{LSB}\left(d_{i}\right) .
$$

Applying the seven round functions changes these to $\left(a_{i}, b_{i}, e_{i}, f_{i}\right)$, the swap to $\left(e_{i}, f_{i}, a_{i}, b_{i}\right)$, and the last round to $\left(e_{i}, f_{i}, g_{i}, h_{i}\right)$. See Figure [6. Note that $K_{18}$ and $K_{19}$ are no longer part of the post-whitening key, but constitute the round key for round eight.

Set $x_{i}=G_{1}\left(e_{i}\right)+G_{2}\left(f_{i}\right)$ and $y_{i}=G_{1}\left(e_{i}\right)+2 G_{2}\left(f_{i}\right)$. For every key-dependent $k$-word vector $S$ we know a mapping from the $2^{64}$ pairs $\left(e_{i}, f_{i}\right)$ to the pairs 


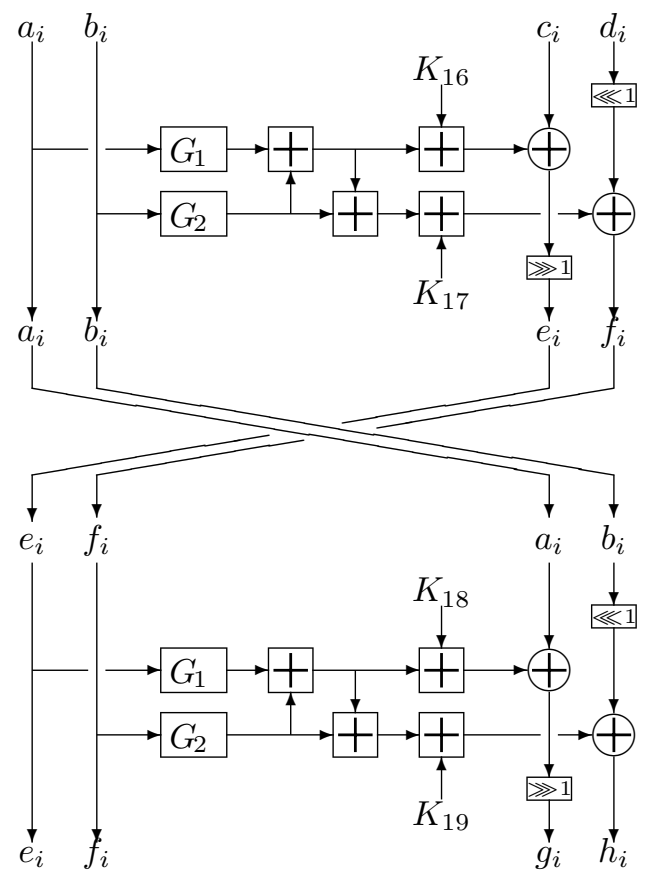

Fig. 6. Round Seven and Eight Without Post-Whitening.

$\left(x_{i}, y_{i}\right)$. So we can virtually blow up the $2^{127}$ ciphertext quadruples $\left(e_{i}, f_{i}, g_{i}, h_{i}\right)$ to six-tuples $\left(e_{i}, f_{i}, x_{i}, y_{i}, g_{i}, h_{i}\right)$. Computing the mapping requires about the time of $2^{64}$ one-round decryptions.

The attack on eight rounds of Twofish works quite similarly to the previous attack. We decompose $\sigma^{*}$ into key-dependent bits $\delta_{a}^{*}\left(K_{18}\right), \delta_{a}^{* \mathrm{c}}\left(K_{18}\right), \delta_{b}^{*}\left(K_{19}\right)$ and $\delta_{f}$ with

$$
\begin{aligned}
\delta_{a}^{*}\left(K_{18}\right) & =\bigoplus_{i}\left(\operatorname{LSB}^{1}\left(G_{1}\left(a_{i}\right)\right)\right)=\bigoplus_{i}\left(\operatorname{LSB}^{1}\left(G_{1}\left(\left(x_{i}+K_{18}\right) \oplus\left(g_{i} \lll 1\right)\right)\right)\right), \\
\delta_{a}^{* \mathrm{c}}\left(K_{18}\right) & =\bigoplus_{i}\left(\operatorname{LSB}\left(G_{1}\left(a_{i}\right)\right)\right)=\bigoplus_{i}\left(\operatorname{LSB}\left(G_{1}\left(\left(x_{i}+K_{18}\right) \oplus\left(g_{i} \lll 1\right)\right)\right)\right), \\
\delta_{b}^{*}\left(K_{19}\right) & =\bigoplus_{i}\left(\operatorname{LSB}\left(G_{2}\left(b_{i}\right)\right)\right)=\bigoplus_{i}\left(\operatorname{LSB}\left(G_{2}\left(\left(\left(y_{i}+K_{19}\right) \oplus h_{i}\right) \ggg 1\right)\right)\right), \\
\text { and } \quad \delta_{f}^{*} & =\bigoplus_{i}\left(\operatorname{LSB}^{1}\left(f_{i}\right)\right) .
\end{aligned}
$$

As above, a subkey triple $\left(\operatorname{LSB}\left(K_{17}\right), K_{18}, K_{19}\right) \in\{0,1\} \times\{0,1\}^{32} \times\{0,1\}^{32}$ is "valid for $S$ " if and only if

$$
\delta_{a}^{*}\left(K_{18}\right) \oplus\left(\delta_{a}^{* \mathrm{c}}\left(K_{18}\right) * \operatorname{LSB}\left(K_{17}\right)\right) \oplus \delta_{b}^{*}\left(K_{19}\right)=\delta_{f}^{*} .
$$


The basic idea for the attack is to run a filtering algorithm to sort out $50 \%$ of the keys, and then to exhaustively search the remaining half of the keys. First we guess the key-dependent vector S, defining $G_{1}$ and $G_{2}$. When $G_{1}$ and $G_{2}$ are known, computing $\delta_{b}^{*}\left(K_{19}\right)$ takes roughly $2^{64}$ very simple steps for each key $K_{19}$. Similarly, computing $\delta_{a}\left(K_{18}\right)$ and $\delta_{a}^{* c}\left(K_{18}\right)$ takes roughly $2^{64}$ very simple steps for each key $K_{18}$. The filtering is significantly faster than exhaustive key search for $K \geq 192$, key finding using this attack takes the equivalent of about $2^{K-2}$ encryptions and hence is twice as fast as exhaustively searching the entire key space.

\subsection{An Improvement for 256-Bit Keys}

Reconsider seven rounds of Twofish and $2^{127}$ plaintexts chosen as before, as described in Section 4.2 and Figure 5 So far, we only have used one distinguishing property: the bits at the LSB-position of the words $d_{i}$ are balanced. Thus we could filter out half of the keys. Using the second property too, we can filter out $75 \%$ of the keys: the bits at the MSB-position of the $c_{i}$-words are balanced.

Set $u_{i}=G_{1}\left(w_{i} \oplus K_{18}\right)+G_{2}\left(x_{i} \oplus K_{19}\right)$ and $v_{i}=u_{i} \oplus K_{16}$. Then $c_{i}=$ $v_{i} \oplus\left(\left(y_{i} \oplus K_{20}\right) \ggg 1\right)$. The bits at the MSB-position of $c_{i}$ are balanced, i,e.,

$$
\bigoplus_{0 \leq i<2^{127}} \operatorname{MSB}\left(c_{i}\right)=0 \Longleftrightarrow \bigoplus_{0 \leq i<2^{127}} \operatorname{MSB}\left(v_{i}\right)=\bigoplus_{0 \leq i<2^{127}} \operatorname{MSB}\left(y_{i} \lll 1\right) .
$$

Consider running the following algorithm:

0 . Choose $2^{127}$ plaintexts as before and ask for the ciphertexts $\left(w_{i}, x_{i}, y_{i}, z_{i}\right)$.

1. For all $(W, X) \in\left(\{0,1\}^{32}\right)^{2}$ : count $\left(\bmod 2^{32}\right)$ the ciphertexts $\left(W, X, y_{i}, z_{i}\right)$.

2. Evaluate $\delta_{y}^{* *}$ by counting the bits $\operatorname{MSB}\left(y_{i} \lll 1\right)$

3. Guess the key-dependent vector $S$, defining the functions $G_{1}$ and $G_{2}$.

4. For each of the $2^{64}$ key pairs $\left(K_{18}, K_{19}\right)$, and every $u_{i} \in\{0,1\}^{32}$ :

Use the $2^{64}$ counters from Step 1 to compute counters $\operatorname{cnt}\left[u_{i}, K_{18}, K_{19}\right]$ for the values $u_{i}=G_{1}\left(w_{i} \oplus K_{18}\right)+G_{2}\left(x_{i} \oplus K_{19}\right)$.

5 . For each of the $2^{32}$ keys $K_{16}$, compute

$$
\delta_{v}^{* *}\left(K_{18}, K_{18}, K_{20}\right)=\bigoplus_{i} \operatorname{MSB}\left(\left(u_{i}+K_{16}\right) * \operatorname{cnt}\left[u_{i}, K_{18}, K_{19}\right]\right) .
$$

6. The triple $\left(K_{16}, K_{18}, K_{19}\right) \in\left(\{0,1\}^{32}\right)^{3}$ is "good for $S$ " if and only if

$$
\delta_{v}^{* *}\left(K_{18}, K_{18}, K_{20}\right)=\delta_{y}^{* *} .
$$

Similar to Section 4.2, the above algorithm requires $2^{127}$ chosen plaintexts, and each of the steps 12 requires $2^{127}$ very simple operations (incrementing a 32 -bit counter). Step 4 requires to guess $K / 2$ key bits, and both the steps 4 and 5 are repeated $2^{K / 2}$ times. Step 4 deals with $2^{64}$ counters and 2 key words, and step 5 deals with $2^{32}$ values $u_{i}$ and 3 key words. Hence, both steps require $2^{128}$ very simple operations. 
The entire algorithm requires about $2 * 2^{(K / 2)+64+64}=2^{(K / 2)+129}$ "very simple" operations and is clearly faster than exhaustive key search for $K \geq 256$.

The same technique works for the attack on eight rounds of Twofish without post-whitening, too. So for $K=256$ a key finding attack based on checking subkeys being both valid and good requires computation time equivalent to about $2^{K-3}$ Twofish encryptions.

\subsection{Finding the Key: Summary}

In Table 2 we summarise our key finding attacks and compare them with exhaustive key search. Note that the running time depends on the number $K \in\{128,192,256\}$ of key bits, and we use one encryption as unit of time.

Table 2. Key Finding Attacks for Twofish.

\begin{tabular}{|c|c|c|c|c|c|c|}
\hline \multirow[t]{2}{*}{ Attack } & \multirow[t]{2}{*}{ Plaintexts } & \multirow[t]{2}{*}{ Rounds } & \multirow{2}{*}{$\begin{array}{l}\text { Key } \\
\text { Bits }\end{array}$} & \multicolumn{2}{|c|}{ Whitening } & \multirow{2}{*}{$\begin{array}{c}\text { Running } \\
\text { Time }\end{array}$} \\
\hline & & & & Pre- & Post- & \\
\hline Section 4.2 & $2^{127}$ chosen & 7 & $K \geq 128$ & yes & yes & $2^{K-2}$ \\
\hline Section $4.2 / 4.4$ & $2^{127}$ chosen & 7 & $K=256$ & yes & yes & $2^{K-3}$ \\
\hline Section 4.3 & $2^{127}$ chosen & 8 & $K \geq 192$ & yes & no & $2^{K-2}$ \\
\hline Section $4.3 / 4.4$ & $2^{127}$ chosen & 8 & $K=256$ & yes & no & $2^{K-3}$ \\
\hline ex. key search & $\approx 3$ known & any & any & any & any & $2^{K-1}$ \\
\hline
\end{tabular}

Note that our attacks are only 2-4 times faster than exhaustive key search, because we filter out $50 \%$ or $75 \%$ of the keys. The overwhelming part of the running time is needed to exhaustively search the remaining key space. Improved distinguishers for six rounds of Twofish, filtering out more keys, could speed up our attacks. Finding such improved distinguishers remains an open problem.

\section{Conclusion}

At present, this paper describes the best known attack on the AES finalist Twofish. We can break up to 8 rounds of Twofish with one-sided whitening faster than exhaustively searching the key would require, though our attacks are only 2-4 times faster than exhausive key search. Since Twofish is a 16-round cipher with twosided whitening, we are able to penetrate exactly one half of Twofish. This still leaves Twofish with a reasonable security margin. An interesting side-note is that the one-bit rotates in Twofish appear to be useful - a variant of without the rotates would allow some enhancement of our attacks.

Also, the paper demonstrates the usefulness of saturation attacks for attacking ciphers which are not square-like. It would be interesting to use saturation techniques to attack other cryptographic primitives. 
Acknowledgements. Supported by Counterpane and Hi/fn, the author participated at the "Third Twofish Retreat" in Utah, USA. He thanks the workshop participants for their encouragement in considering saturation attacks for Twofish. Also, the FSE referees provided useful comments to improve this presentation.

\section{References}

1. E. Biham, A. Biryukov, A. Shamir, "Miss in the Middle Attacks on IDEA and Khufru", Fast Software Encryption 1999, Springer LNCS 1636, pp. 124-138.

2. E. Biham, "Cryptanalysis of Ladder DES", Fast Software Encryption 1997, Springer LNCS 1267, pp. 134-138.

3. C. D'Halluin, G. Bijnens, V. Rijmen, B., Preneel, "Attack on six round of Crypton", Fast Software Encryption 1999, Springer LNCS 1636, pp. 46-59.

4. J. Daemen, L. Knudsen, V. Rijmen: "The block cipher Square", Fast Software Encryption 1997, Springer LNCS 1267, pp. 149-165.

5. J. Daemen, V. Rijmen, "AES proposal: Rijndael" (2nd version) 18.

6. N. Ferguson, "Impossible differentials in Twofish", Twofish TR \#5, 1999 [18].

7. N. Ferguson, J. Kelsey, S. Lucks, B. Schneier, M. Stay, D. Wagner, D. Whiting, "Improved Cryptanalysis of Rijndael" Fast Software Encryption 2000, to appear.

8. J. Kelsey, "Key Separation in Twofish", Twofish TR \#7, 2000 [18.

9. L. Knudsen, "Truncated and Higher Order Differentials", Fast Software Encryption 1995, Springer LNCS 1008, pp. 196-211.

10. L. Knudsen, "Trawling Twofish (revisited)" May 15, 2000, 18.

11. C. H. Lim, "Crypton: a new 128-bit block cipher" 18.

12. C. H. Lim, "A revised version of Crypton - Crypton V1.0 - ", Fast Software Encryption 1999, Springer LNCS 1636, pp. 31-45.

13. F. Mirza, S. Murphy, "An Observation on the Key Schedule of Twofish", In: The 2nd Advanced Encryption Standard Conference, pp. 151-154, April 1999 [18].

14. S. Murphy, "The Key Separation of Twofish", 2000 [18].

15. B. Schneier, J. Kelsey, D. Whiting, D. Wagner, C. Hall, N. Ferguson, "Twofish: A 128-bit Block Cipher" [18.

16. B. Schneier, J. Kelsey, D. Whiting, D. Wagner, C. Hall, N. Ferguson, "The Twofish Encryption Algorithm", Wiley, 1999.

17. "The GNU Privacy Guard", "http://www.gnupg.org/".

18. "AES Development Effort", NIST, "http://www.nist.gov/aes". 